\title{
Bus Protection in Ship MVDC Power System
}

\section{Yangting Xiao, 2,a , Zhihao Ye ${ }^{1, b}$, Muyi Yin ${ }^{3, c}$, Yuzhou $\mathrm{Hu}^{2, \mathrm{~d}}$}

\author{
${ }^{1}$ National Key Laboratory and Technology on Vessel on Integrated Power System, Naval University \\ of Engineering, Wuhan, 430033, China
}

${ }^{2}$ Shanghai Marine Equipment Research Institute, Shanghai, 200031, China

${ }^{3}$ Wuhan Ordnance N.C.O. Academy, Wuhan, 430075, China

aemail:13780213@qq.com, bemail:yxyx928@126.com, cemail:923634866@qq.com, demail:hyz0404@126.com

\begin{abstract}
Keywords: Shipboard Power System; Bus-bar protection; Bus-bar Differential Relay; Dead zone; Medium Voltage DC System
\end{abstract}

\begin{abstract}
Bus differential protection is effective measures to cope with the bus-bar fault in ship MVDC system. A realization of DC bus-bar protection is based on digital protection device which are suitable for complex medium voltage power system. Firstly, the principle of DC bus-bar differential protection, the action characteristic and the connection mode of current transmitter are introduced. After that the dead zone of DC bus-bar protection and its counter measures are analyzed. Finally, the measures to increase the reliability of DC bus-bar protection are discussed according to the special environment of shipboard power system. The analysis results can be used as a reference for Ship MVDC power system protection design.
\end{abstract}

\section{Introduction}

Currently, the capacity of the marine electric equipment increase greatly, many large ships and ocean engineering equipment have been used in medium voltage, and try to operate with direct current system, wherein the system structure is becoming different and require continuous power supply. Correspondingly, the Marine Power System Protection require more reliability, speed and selectivity $[1,2]$. In Ship MVDC power systems, the DC bus-bar short-circuit failure is small probability event, but it cause great harm to power system, may lead to arcing fault inside the switchboard. There are many reasons resulting in DC bus-bar short-circuit, including insulator aging, voltage or current transmitter failure, a plague of rats. When the DC bus-bar short-circuit occurs and continues for a long time, an internal arc fault will continue burning and release tremendous energy, damage the device within the switchboard and endanger crew life.

For DC bus-bar short-circuit, there are three kinds of protection method, including the arc protection, short delay over-current protection, DC bus-bar protection [3, 4]. First, the arc protection is a sensitive and rapid form of protection [3], relying on optical probe to detect arcing inside the switchboard. But the setting of arc protection is relatively difficult, when the fault locate in the dead zone, it is difficult to fit with the adjacent area protection, the entire set of validation tests are difficult to carry out. The second approach is based on the short time delay which is one of three-stage over-current protection [4]. This approach will generally result in continued arcing hundreds of milliseconds before fault trip; it is only as a back-up protection. The third way is to configure a special DC bus fault protection, which is based on computer protection and using differential relays. As a form of protection, it has the advantage of fast, selective. And in ship rectifier generator protection, circuit protection, ground fault protection, the differential relay has accumulated a wealth of operating experience, but differential protection does not apply in the DC bus-bar protection.

This paper argues that in the MVDC ship power systems, DC bus differential protection can be used as a main protection against DC bus-bar fault, and proposed a new DC bus-bar protection system implemented by computer protection device for ship MVDC power system. Firstly, this paper introduced the principle and operating characteristics of DC bus differential protection. 
Secondly, by analyzing the wiring of protection method, this paper focuses on the dead DC bus protection and remedies, then discuss the cooperation logic with other protection methods. Finally, the special environment of a ship power system, this paper discusses effective measures to improve the reliability of the DC bus-bar protection.

\section{DC bus differential protection principle}

Different from the differential protection of the line and rectifier generator [3, 5], the input current of DC bus-bar differential protection is more, as shown in figure 1(a). If the predetermined direction of the current flowing into the DC bus as a positive direction, the differential relay can calculate phases sum of all the current inflow DC bus-bar, as a differential current or action current $I_{d}$, calculate scalar sum as braking current $I_{r}$, DC bus differential protection operating characteristic as represented by the Eq. 1 .

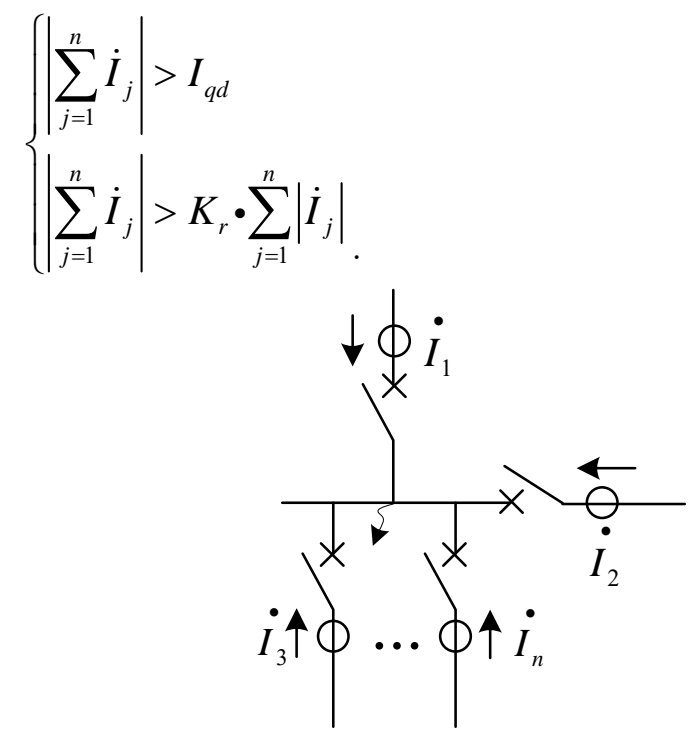

(a) wiring diagram



(b) the operating characteristics

Figure 1 Bus-bar differential protection wiring diagram and the operating characteristics

In formula, $\dot{I}_{j}$ i s current inflow DC bus, $I_{q d}$ is start threshold, $K_{r}$ is ratio braking coefficient, DC bus differential relay operating characteristic shown in Figure 1(b), where the shaded area is the operation area.

Because of $\left|\sum_{j=1}^{n} \dot{I}_{j}\right|>\sum_{j=1}^{n}\left|\dot{I}_{j}\right|$, the area above the action curve whose slope is equal to 1 in Figure 1(b) is outside the defined domain. When DC bus-bar normal operation and fault occurs in the external area, the current that flows into the DC bus bar should be equal to the current outflow the DC bus. When the transmission error of the current transmitter (referred to as CT) is not considered, the action current of the differential relay is 0 . In the actual situation, the CT characteristic of each connecting element is different, the transmission error constitutes the differential current, so the action threshold Iqd and the ratio braking characteristic $\mathrm{Kr}$ are being set. When a fault occurs on the DC bus, the connecting unit current flows into the DC bus-bar, and the action current of the differential relay is the short-circuit current at the fault point, and the differential protection is reliable. As a unit protection, the protection range of the differential protection is defined by the CT, so the CT is generally located in the adjacent unit area of the circuit breaker.

The application of the above mentioned principle in the actual ship electric power system is shown in Figure 2. Figure 2 is a part of typical ship power system single line diagram, two rectifier generators each with independent DC bus connected by coupling QF-M. First, considering the fault occurring at three different location, such as the left DC bus F-A1, the right DC bus F-A2 and line 
F-X in Figure 2. When the fault occurs in the left DC bus F-A1, the current of CT-G1, M, X, L1 flow in differential relay R1, R1 will judge the internal fault an F-A1(simulation waveform see Figure 3(a)), then braking QF-G1, M, X, L1. The current of CT-G2, M, L2 flow in differential relay R2, R2 will judge the external fault. Similarly, when the fault occurs at F-A2, R2 will judge the internal fault, then braking QF-G2, L2, M. When the fault occurs at F-X(simulation waveform see Figure 3(b)), R1 and R2 will judge the external fault at the same time, the DC bus protection will not action. Need to be explained, F-A1, A2, X is three fault location more likely to occur, but it can't cover all areas inside or outside the switchboard, it will be to be analyzed in detail in the next section.

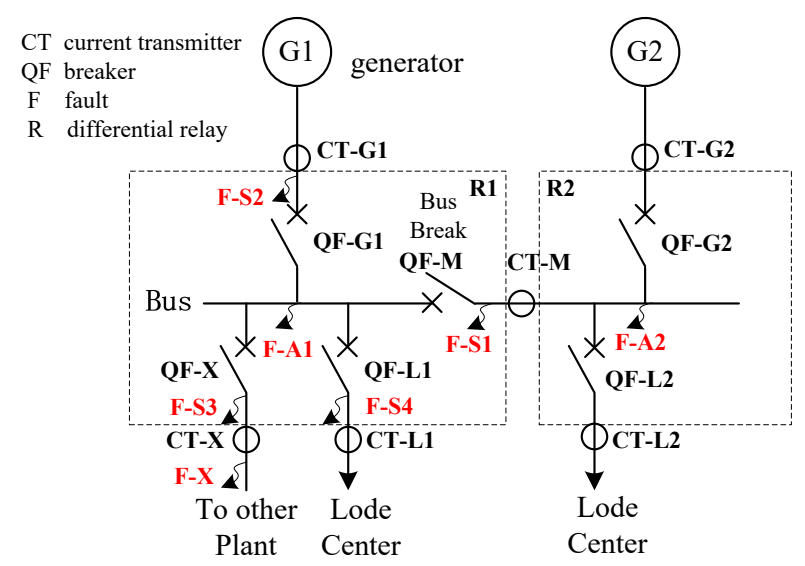

Fig.2. Principle diagram of DC bus-bar protection

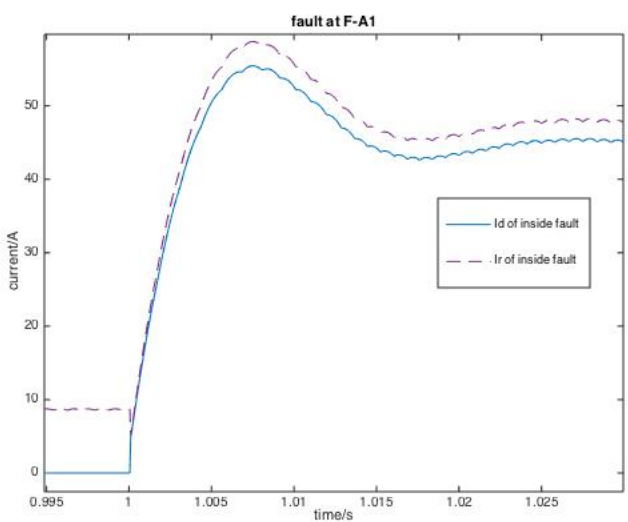

(a) fault at F-A1

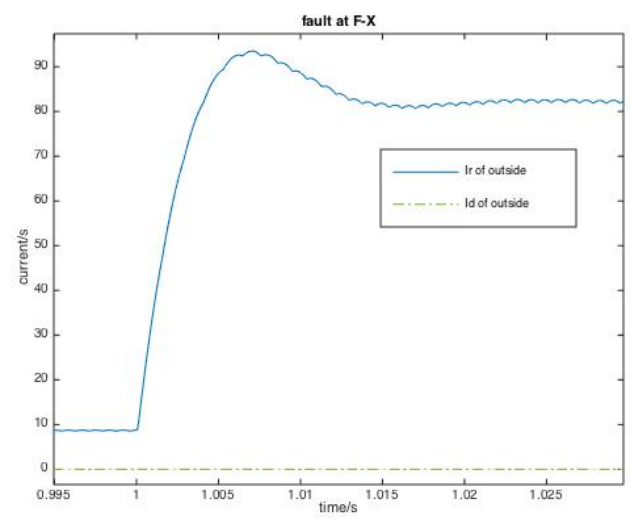

(b) fault at F-X

Fig.3. simulation waveform

\section{Dead zone of DC bus-bar protection and Countermeasures}

In the ship MVDC system, the input analog quantity of the protection device is collected by the current transmitter CT, and the breaking fault is realized by the medium voltage circuit breaker QF. Due to the layout of switchboard, there is a short DC bus between CT and QF inevitably, As shown in Figure 1(b), F-S1, S2, S3, S4 premises. When these positions are in fault, the single differential relay $\mathrm{R} 1$ and $\mathrm{R} 2$, which is described in the last section, will fail to cover the fault location.

(1) DC bus dead zone fault

Assumed that the fault occurred in the DC bus at F-S1, the minimum fault isolation method is breaking the QF-G2, L2, M. As Figure 1(b) shows, in the closure of the DC bus-bar QF-M, Electrical characteristics of the fault at $\mathrm{S} 1$ and $\mathrm{A} 1$ are exactly the same, differential relay $\mathrm{R} 1$ will be sentenced to the left side of the fault, broken QF-G1, X, L1, M. The actual fault occurs on the right DC bus fault is not removed. At the same time, the scope of protection relay R2 can't cover F-S1, it always sentenced to an external fault, so that F-S1 is dead zone.

The solution of F-S1 fault dead zone need to be supplemented by the DC bus-bar dead zone protection, its action logic is as follows: after DC bus fault, X, L1, M, QF-G1 are disconnected by 
the differential relay R1, the transmitter CT-M always has fault current flowing through. At this time, differential relay $\mathrm{R} 1$ and the over-current relay detecting DC bus CT-M will not return. Protection device can accurately determine the fault is located in the dead zone, immediately disconnect the QF-G2, L2, M in order to remove the fault. At this time the scope of the accident is expanded to the whole power plant. The setting time $T_{\mathrm{sq}}$ of dead zone protection start delay when the DC bus protection act, and avoid the circuit breaker breaking time and over-current protection element return time, as shown in Eq. 2.

$$
t_{s q}=\left(t_{2}+t_{3}\right) \cdot k \text {. }
$$

In formula, $t_{2}$ is the circuit breaker breaking time, $t_{3}$ is the return time required for the Fourier data window of the over-current element, and $\mathrm{K}$ is the safety factor, as shown in Figure $4 . t_{2}$ is the inherent parameters of circuit breaker, $t_{3}$ can be reduced by using a shorter time window algorithm.

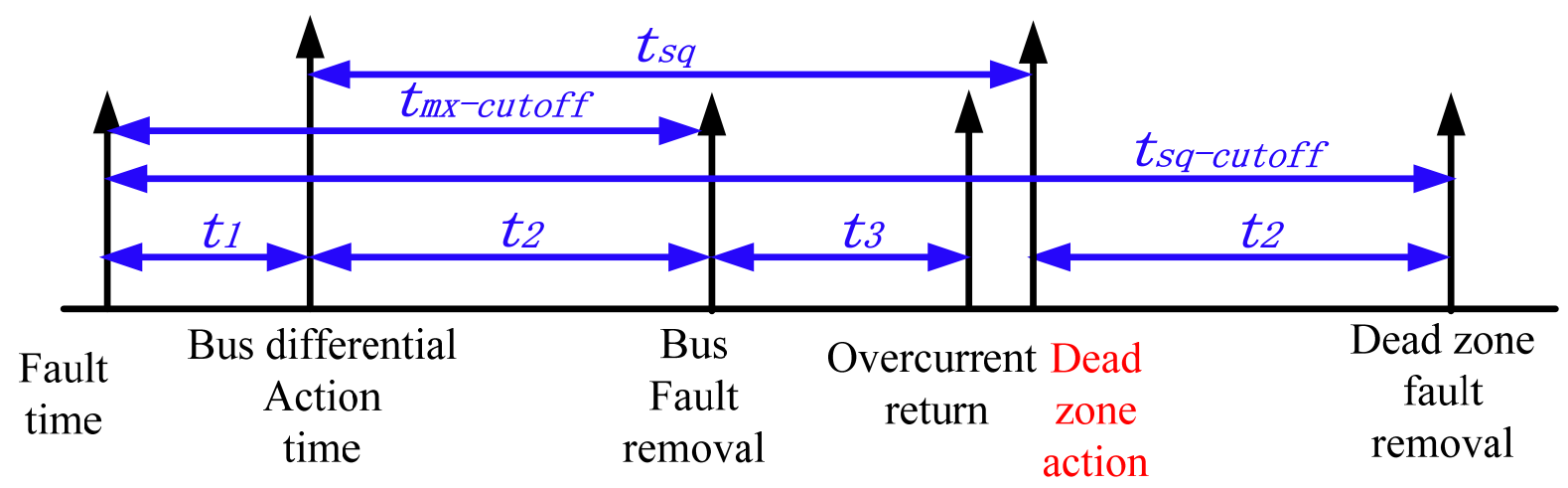

Fig.4. DC bus protection action sequence diagram

Show as Figure $4, t_{m x-\text { cutoff }}$ is the last DC bus fault clearing time, $t_{s q-\text { cutoff }}$ is the last dead zone fault clearing time, respectively show as Eq. 3, Eq. 4, the $\mathrm{T} 1$ is the $\mathrm{DC}$ bus differential protection action time.

$$
\begin{aligned}
& t_{\text {mx-cutoff }}=t_{1}+t_{2} . \\
& t_{\text {sq-cutoff }}=t_{1}+t_{s q}+t_{2}=t_{1}+\left(t_{2}+t_{3}\right) \cdot k+t_{2} .
\end{aligned}
$$

Need to be explained, If the differential relay R1, R2 is realized by two sets of device, then dead zone protection device need output signal to the other a protection device to tripping circuit breaker associated with the second differential relay. The DC bus protection device should collect the secondary side current of G2, X, CT-G1 L1, L2, M at the same time, and the differential relay R1, $\mathrm{R} 2$ located in the same device to increase the reliability of protection system.

(2) Dead zone fault of other position

If the fault occurs in the rectifier generator switchboard dead zone F-S2, because the QF-G1 has been broken by the differential relay $\mathrm{R} 1$, it is only necessary to send de-excitation signal to the rectifier generator G1. If the fault occurs in the line F-S3, the line differential protection will be judged as an external fault. After the DC bus differential relay R1 cutting off line switch QF-X, CT-X is still over current. If the fault occurs in the load switchboard dead zone F-S4, due to on power in the load side, L1 trip off to remove the fault. When troubleshooting, it is time to clean up the fault in dead zone.

In summary, the protection action of all kinds of DC bus-bar fault is summarized as shown in table 1.Because of the dead zone between the transmitter CT and the circuit breaker QF, protection devices should be equipped with dead zone protection and join with the adjacent area protection. 
Table $1 \quad$ DC bus protection under all kinds of situation

\begin{tabular}{|c|c|c|c|c|}
\hline $\begin{array}{c}\text { Fault } \\
\text { location }\end{array}$ & description & Action breaker & $\begin{array}{c}\text { Minimum fault } \\
\text { isolation method }\end{array}$ & Action relay \\
\hline A1 & Left bus (In) & G1、L1、M、X & G1、L1、M、X & R1 \\
\hline A2 & Right bus (out) & G2、L2、M & G2、L2、M & R2 \\
\hline X & line (out) & X break by line & X break by line & $\begin{array}{c}\text { Line protection } \\
\text { action }\end{array}$ \\
\hline S1 & Bus dead zone & $\begin{array}{c}\text { G1、L1、M、X } \\
\text { G2、L2、 }\end{array}$ & G2、L2、M & $\begin{array}{c}\text { R1、 dead zone } \\
\text { protection }\end{array}$ \\
\hline S2 & Gen dead zone & G1、L1、M、X & G1 & $\begin{array}{c}\text { R1、 dead zone } \\
\text { protection }\end{array}$ \\
\hline S3 & Line dead zone & G1、L1、M、X & $\begin{array}{c}\text { R1、 dead zone } \\
\text { protection }\end{array}$ \\
\hline S4 & Lode dead zone & G1、L1、M、X & L1 & $\begin{array}{c}\text { R1、 dead zone } \\
\text { protection }\end{array}$ \\
\hline
\end{tabular}

\section{Measures to increase the reliability of DC bus-bar protection}

As the ship power system power flow convergent point, DC bus is connected to the rectifier generator and load, need to join the measures to improve the reliability of the DC bus protection, to prevent removing DC bus without faults leading to loss of power of the large area of the ship power system. Electronic Current transmitter as an important part of the whole system should become the focus of attention.

(1) Measures to deal with CT saturation

In DC system, electronic power sensor will appear saturation problem, but it's different from the saturation characteristics of AC electromagnetic; when the current is out of measuring range, DCCT directly cut off the value cannot be measured. So in parallel system, when a large range CT measures accuracy, and the small range CT being in saturation and cutoff, the Calculation result of action current will be much great, DC bus protection may start up and trip the breaker. So, the ratio break coefficient should be calculated at the special application background, avoid the phenomenon of false action caused by saturation. Another way to avoid of CT saturation is choosing Optical current transducer, which can measure a wide range of current, much larger than the fault current of the system.

(2) Measures to deal with CT disconnection

In DC bus protection device, the $\mathrm{CT}$ disconnection detecting element should be set to increase the reliability. When the system runs normally and CT disconnection occurs, the differential current of the broken line is the load current of the line, so the proportional brake starting current of the differential element is in the maximum load current of each component.

\section{Conclusion}

This paper presents a DC bus protection system suitable for the medium voltage ship power system, analyzes the DC bus differential protection principle, operation characteristics, protection of wiring, and discusses the matters needing attention when using DC bus protection:

(1) The CT for DC bus differential protection using should be located in the area adjacent to the outer side of the circuit breaker. The area between the circuit breaker and the transmitter is called the dead zone. Protection device should be equipped with dead zone protection, and join with the adjacent protected areas of the logic.

(2) In the design of protection, pay attention to prevent CT saturation, CT disconnection, improving the reliability of DC bus protection.

Through appropriate precaution and reasonable configuration, DC bus protection system can be selectivity, fast and reliability; it will be the main protection of fault on the ship MVDC system. The next step should be processed from the ship's own needs and inherent characteristics, considering 
the special environment of the ship, carry out a large number of basic works from the data collection, protection of information standardization and other aspects. The results of this paper can be used as a reference for the protection design of the ship MVDC power system.

\section{References}

[1] ZHAO Yue-ping, WANG Liang-xiu. The Development and Application of Ship Electric Technology[J] Shanghai Shipbuilding No.4, 2008.

[2] MO Wenke, XU Binhai. Analysis on Key technology for Design of Ship Medium Voltage AC Supply System [J]. Ship Engineering. Vol.35 2013,S1.

[3] Tian Guangqing. The Arc Protection System and Its Application to the Internal Fault of LV/MV Switchgear and the Busbar [J]. Electrotechnical Journal. No.1,2004.

[4] XU Binhai and so on. Analysis on Protective Scheme for Mass Generators Shipboard Supply Power System[J], Mechanical and Electrical Equipment. No.3,2013.

[5] Cao Shuang, Wang Qingyu, Zhang Jiyuan. Longitudinal Differential Protection in Marine Power System [J]. Marine Electric \& Electronic Engineering.Vol.27,No.2, 2007.3/4.

[6] HAN Qi, WANG Demin, MO Wenke. Application of Zero-Sequence Differential Protection in Ship Power System [J]. Ship Engineering. Vol.37, No.10, 2015.

[7] Zhu Shengshi. Principle and technology of high voltage power network relay protection[M]. CHINA ELECTRIC POWER PRESS, 2012.

[8] YU Jiang and so on. Improvement of 500kV Breaker Failure and Dead-zone Prevention Based on Requirement on Stability of Grid [J]. Automation of Electric Power Systems. Vol.39 No.2 Jan.25, 2015. 